\title{
Fast-rotating nearby solar-type stars ${ }^{\star} \star \star$
}

\section{Li abundances, $v$ sin $i$ and X-ray luminosities relationships}

\author{
G. Cutispoto ${ }^{1}$, G. Tagliaferri ${ }^{2}$, J. R. de Medeiros ${ }^{3}$, L. Pastori $^{2}$, L. Pasquini ${ }^{4}$, and J. Andersen ${ }^{5}$ \\ 1 INAF - Catania Astrophysical Observatory, v. S. Sofia 78, 95125 Catania, Italy \\ e-mail: gcutispoto@ct.astro.it \\ 2 INAF - Brera Astronomical Observatory, via Bianchi 46, 22055 Merate (LC), Italy \\ e-mail: pastori@merate.mi.astro.it; gtagliaf@merate.mi.astro.it \\ 3 University Federal of Rio Grande do Norte, Department of Physics, 59072-970 Natal, R.N., Brazil \\ e-mail: renan@dfte.ufrn.br \\ ${ }^{4}$ European Southern Observatory, Karl-Schwarzschild-Strasse 2, 85748 Garching bei München, Germany \\ e-mail: lpasquin@eso.org \\ 5 Astronomical Observatory, NBIfAFG, Juliane Maries Vej 30, 2100 Copenhagen, Denmark \\ e-mail: ja@astro.ku.dk
}

Received 8 July 2002 / Accepted 28 October 2002

\begin{abstract}
We present an analysis of our high-resolution spectroscopic and high-precision $U B V(R I)_{\mathrm{c}}$ photometric observations of a sample of 110 nearby late-F and G-type stars selected for their large rotational velocity. The relationships between Li abundance, X-ray luminosity, and $v \sin i$ are investigated. We find that, as expected, the stars in our sample show statistically higher $\mathrm{Li}$ abundance and activity level than field star samples with similar characteristics, but slower rotation. Surprisingly, however, we also find four rapidly-rotating single main-sequence stars with very low $\mathrm{Li}$ abundance. For both single and binary stars we find a large spread of Li abundance for stars with rotation lower than about $18 \mathrm{~km} \mathrm{~s}^{-1}$. The well-established correlation between $\mathrm{X}$-ray luminosity and rotation rate is clearly observed. All single unevolved solar type stars with $v \sin i$ larger than $18 \mathrm{~km} \mathrm{~s}^{-1}$ are strong X-ray emitters and have high Li abundance. Finally, we find also five evolved stars with very low Li abundance that are still rather fast rotators. The results from our sample confirm the presence of young very active stars close to the Sun, in agreement with recent findings from EUV and X-ray surveys, although our sample does not show such extreme characteristics as those selected from EUV and X-ray surveys at the current flux limits.
\end{abstract}

Key words. stars: abundances - stars: activity - stars: fundamental parameters - stars: variables: general - X-rays: stars

\section{Introduction}

One of the main topics in stellar astrophysics is to explain how the non-classical phenomena observed on the Sun and solartype stars depend on the main stellar parameters, particularly the magnetic and dilution processes responsible for stellar activity. In practice, such studies focus on the connection between rotation, magnetic activity diagnostics and light element (in particular $\mathrm{Li}$ ) abundances, and on the dependence of any such connections on metallicity, mass and age. In low-mass main sequence (MS) stars, internal structure is determined

Send offprint requests to: G. Cutispoto,

e-mail: gcutispoto@ct.astro.it

* Based on data collected at the European Southern Observatory, La Silla, Chile.

$\star \star$ Tables, Figures and the complete data set are available in electronic form at the CDS via anonymous ftp to cdsarc.u-strasbg.fr $(130.79 .128 .5)$ or via

http://cdsweb.u-strasbg.fr/cgi-bin/qcat?J/A+A/397/987 primarily by stellar mass rather than age. In contrast, surface activity as manifested in X-rays, at least for late-type dwarfs, seems to scale directly with rotation and by consequence with age, but is only slightly dependent on mass (Pallavicini et al. 1981; Schmitt et al. 1985; Hempelmann et al. 1995; Stauffer et al. 1997). This fact would seem to result from the significant decrease in rotation, due to the magnetic torque from a magnetically-coupled stellar wind.

Hence, we believe that it would be extremely interesting to study a sample of stars selected directly on the basis of high rotational velocity rather than from such parameters as high (X-ray) activity or young age. We would expect such a sample to be composed, essentially without exception, by stars with high coronal activity, including both young single (or binary) stars as well as short-period older binaries. The former should then have a very high $\mathrm{Li}$ abundance (i.e. higher than the Hyades), the latter a large spread of $\mathrm{Li}$ abundance. In any case, the average $\mathrm{Li}$ abundance should be higher than that expected in field stars of the same spectral type, selected only on the 
basis of their distance from the Sun. We stress that in such a sample we would not expect to find neither low-activity stars nor single stars with low Li abundance.

There are several reasons to expect that our sample will show higher Li abundances than randomly selected star samples with similar spectral type. For single stars the reason is that, although a strict one-to-one correlation does not hold, it has been shown that young stars tend to have higher Li abundances than old stars (see the discussion in Pasquini et al. 1997). Hence, because our sample is selected on the basis of fast rotation (i.e. a rotational velocity similar to or higher than the value observed for stars of comparable spectral type in the Hyades), the single stars are expected to be rather young, typically younger than $1 \mathrm{Gyr}$. Therefore, they are expected to show $\mathrm{Li}$ abundance levels similar to or higher than those observed in the Hyades. For binary stars the reason is that the rapid rotators which are not young are likely to be members of tidally locked systems. Although for some of them we expect to find low $\mathrm{Li}$ abundances, it has been recognized that the Li content in such systems is, at least on a statistical basis, larger than in randomly selected stars (Randich et al. 1993, 1994; Fernandez-Figueroa et al. 1993; Barrado y Navascues et al. 1998).

However, we note that it is not clear which is the physical cause for this difference; several theories predict that rotation itself (or the rotational history of the star) may influence the Li depletion through either meridional circulation or rotationally induced mixing (Charbonnel et al. 2000; Pinsonneault et al. 2000). Evidence for some dependence of Li abundance on rotation has been observed among K-type stars in young clusters (Soderblom et al. 1994; Garcia-Lopez et al. 1994) and even among slow rotators in the $\alpha$ Per cluster (Randich et al. 1998). We also need to consider that the clusters in which this rotational dependence of $\mathrm{Li}$ has been observed are all rather young, and stars in such clusters may have rotations up to several tens of $\mathrm{km} \mathrm{s}^{-1}$. Finally, it is not clear if such dependence of $\mathrm{Li}$ on rotation is an artifact due to the use of inappropriate atmospheric models or if phenomena like stellar activity play an important role in this context (cf. Pasquini 2000; Randich 2001; Cutispoto 2002).

A sample of nearby fast-rotating solar-type stars was selected and studied by Cutispoto et al. (2002), hereafter referred to as Paper I. Accurate spectral classifications, effective temperatures $\left(T_{\text {eff }}\right)$, rotational velocities $(v \sin i), \mathrm{Li}$ abundances $\left(A_{\mathrm{Li}}\right)$, radial velocities $(R V)$, and $\mathrm{X}$-ray luminosities $\left(L_{\mathrm{X}}\right)$ were determined. In this paper we discuss the $\log T_{\text {eff }}$ vs. $A_{\mathrm{Li}}$ diagrams, compare the $A_{\mathrm{Li}}$ of the stars of our sample with those of the Pleiades and Hyades clusters and with a sample of stars not selected on the basis of $v \sin i$; we also discuss the relationships between $v \sin i, A_{\mathrm{Li}}$ and $L_{\mathrm{X}}$ and the global properties of our sample.

\section{Sample definition and observations}

In order to identify young solar-type stars in the solar neighborhood we started from the CORAVEL survey of $3200 \mathrm{~F}$ to G-type stars brighter than $V \simeq 8.6$ (Nordström et al. 1999). We defined the sample by selecting all stars with significant rotational velocity as measured from the width of the CORAVEL cross-correlation profile. Specifically, a lower limit of $8 \mathrm{~km} \mathrm{~s}^{-1}$ was chosen for the $\sigma$ of the Gaussian fit to the profile (see Sect. 2.1 in Paper I for further details). This choice ensures that all the selected stars are likely to fulfil one of the signatures for youth, significant rotational velocity.

Among the stars thus selected, 129 can be observed in the southern hemisphere, and we observed them spectroscopically with the 1.4-m ESO CAT telescope, and photometrically with the 50-cm ESO telescope, in various observing runs. For each star we derived an accurate spectral classification and determined $R V, v \sin i, A_{\mathrm{Li}}$ and $L_{\mathrm{X}}$ (see Paper I for details). All data used in the analysis and figures presented in this paper are taken from Table 1 of Paper I.

Out of the 129 stars observed, we found that 19 are not true solar-type stars (see Sect. 3.2 in Paper I), and they are not further studied in this paper. The remaining 110 stars here presented comprise 42 single stars, 33 visual binaries (VB), and 35 spectroscopic binaries (SB). Among the VB components, 35 are not SBs (see Table 1 in Paper I).

In the following analysis we treated the 35 single components of VBs, whether primaries or secondaries, as single stars, because their rotation rate is not influenced by the presence of the companion. Moreover, we were able to obtain separate spectral classifications for 61 of the components of SBs (see Table 1 and Appendix 2 in Paper I for details). In summary, therefore, data for 138 objects, including 77 single stars $(42+35$ as explained above) and 61 close binary components are available for the analysis performed in this paper.

Figure 1a shows the $B-V$ vs. $M_{V}$ colour-magnitude diagram (CMD) for these 138 stars. In this figure we also plot the MS region and the giant sequences determined from Hipparcos data (Houk et al. 1997). Our sample contains a total of 99 MS stars (59 single and 40 close binaries) and 39 non-main sequence stars (18 single and 21 close binaries). It also contains a higher percentage of close binary stars compared to what is expected from a sample of randomly selected field stars. This is expected, not only because the binaries are on average brighter than single stars, but also because our selection criterion (i.e. high rotation rate, see Sect. 4.1 in Paper I) favours the inclusion of tidally synchronised binary stars. In this context, our working sample is one of the most complete samples of solar-type stars studied yet. In Fig. 1b we highlight the CMD diagram for the stars we believe to be very young (see Sect. 3.1).

\section{Results}

\subsection{The $A_{L i}$ vs. $\log T_{\text {eff }}$ diagram: single stars}

Figure $2 \mathrm{a}$ shows the $A_{\mathrm{Li}}$ vs. $\log T_{\text {eff }}$ distribution for MS single stars and MS single components of VBs (a total of 59 stars). The solid and dashed curves are the fiducial $A_{\mathrm{Li}}$ vs. $\log T_{\text {eff }}$ curves for the Pleiades (top envelope) and the Hyades clusters, respectively (adapted from Deliyannis 2000). Figure $2 b$ shows the same distribution, but for the stars above the MS (a total of 18 stars). The area inside the quadrangle is the region populated by subgiants, giants and supergiants (adapted from Mallik 1999). The Sun's position is shown in both panels. 


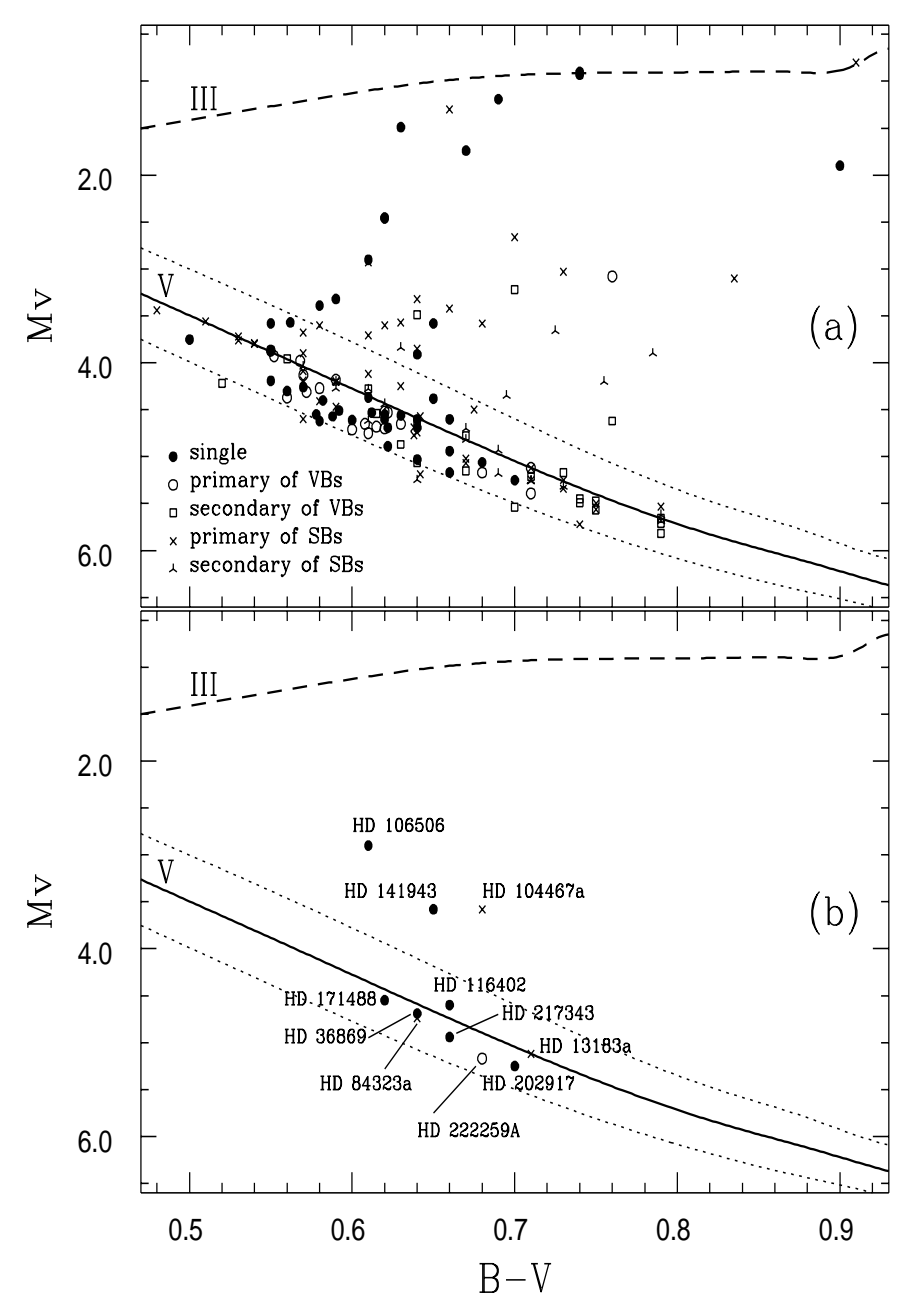

Fig. 1. The $B-V$ vs. $M_{V}$ diagram for the stars in our sample. Single stars and primary and secondary componentes of VBs and of SBs are indentified by different symbols. The continuous line and the longdashed line outline the main sequence and giant regions, respectively, from Hipparcos data (Houk et al. 1997); the short-dashed lines indicate the limits of the dispersion of main sequence stars from Hipparcos data. Panel a) shows the complete sample; panel b) shows the stars we believe to be very young.

Most of our MS single stars are above the Hyades track. We know that stars with low $A_{\mathrm{Li}}$ exist among old, inactive, slowrotating field stars (Pasquini et al. 1994). Intermediate-age stars have comparable, albeit slightly lower $A_{\mathrm{Li}}$, than the Hyades, as confirmed by the observations of $\mathrm{Li}$ in intermediate-age open clusters (Randich et al. 2000), which, however, do not show objects with low $A_{\mathrm{Li}}$ in the colour range we consider. In contrast, a large scatter in $A_{\mathrm{Li}}$ is clearly present in the solar-age open cluster M 67 (Pasquini et al. 1997). In summary, while $A_{\mathrm{Li}}$ is not a good age tracer for stars older than about $1 \mathrm{Gyr}$, it has been shown that stars with $A_{\mathrm{Li}}$ comparable to or above that of the Hyades can be confidently classified as being as young as the Hyades or even younger.

Accordingly, it is immediately clear that many single stars in our sample are indeed quite young, with ages lower than 1 Gyr. In particular, we find that among the single MS stars studied, $6(\simeq 10 \%)$ have an $A_{\mathrm{Li}}$ higher than that of the Pleiades,

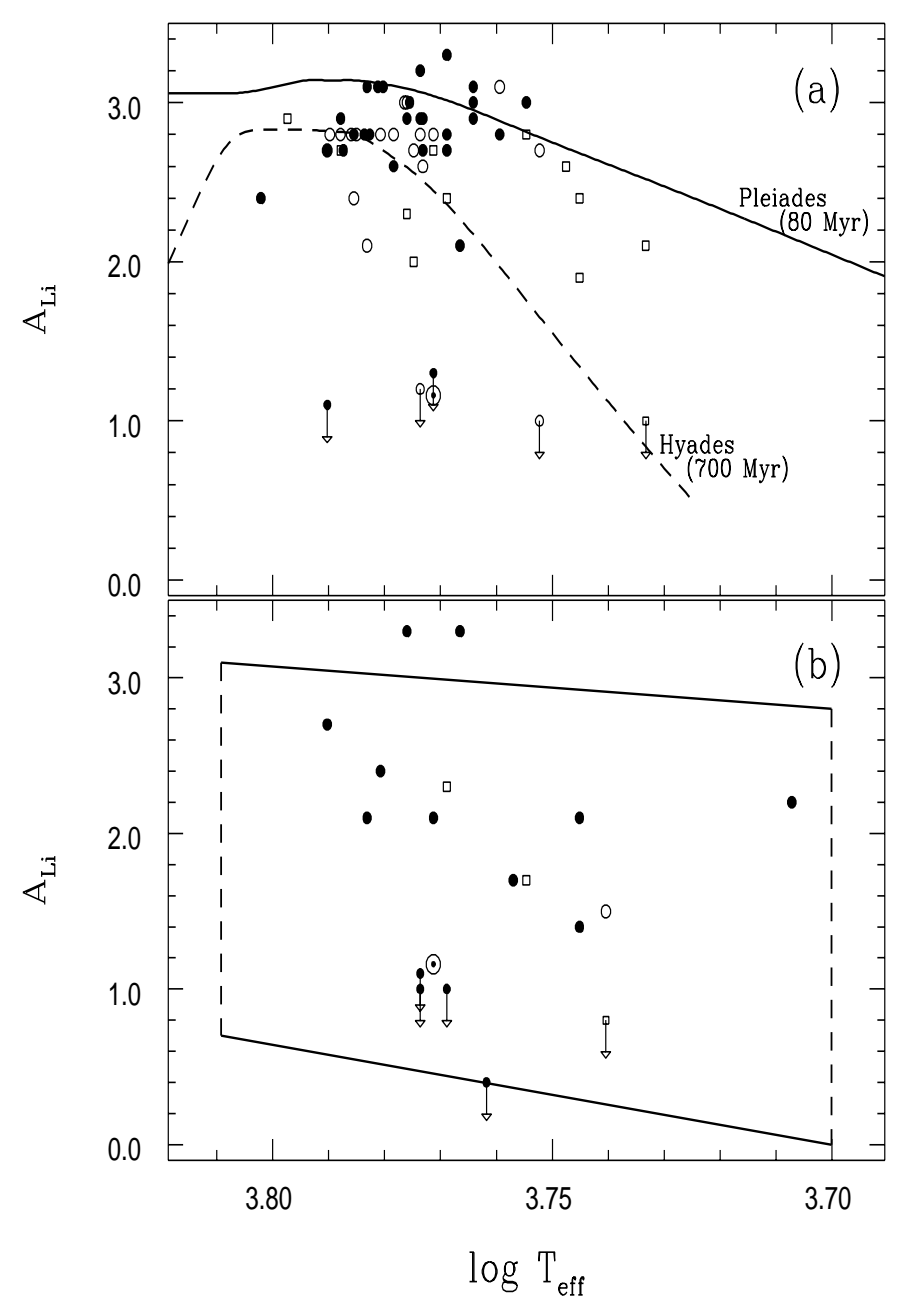

Fig. 2. $A_{\mathrm{Li}}$ vs. $\log T_{\text {eff }}$ for single stars and single VB components. Symbols as in Fig. 1. Panel a) stars on the main sequence; the solid and dashed curves are the fiducial $A_{\mathrm{Li}}$ vs. $\log T_{\text {eff }}$ curves for the Pleiades (top envelope) and the Hyades clusters, respectively (adapted from Deliyannis 2000). Panel b) stars above the main sequence; the area inside the quadrangle is the region populated by subgiants, giants and supergiants (adapted from Mallik 1999). The Sun's position is shown in both panels.

$38(\simeq 64 \%)$ have an $A_{\mathrm{Li}}$ between that of the Pleiades and Hyades, and $10(\simeq 17 \%)$ have an $A_{\mathrm{Li}}$ lower than that of the Hyades, while for only 5 stars $(\simeq 9 \%)$ we were just able to measure an upper limit for $A_{\mathrm{Li}}$. This result can be compared with the observations by Pasquini et al. (1994) of a sample of field stars of similar spectral type, but not selected on the basis of high $v \sin i$. Of the $42 \mathrm{MS}$ single stars they studied, none has an $A_{\mathrm{Li}}$ higher than that of the Pleiades, $10(\simeq 24 \%)$ have an $A_{\mathrm{Li}}$ in between that of the Pleiades and Hyades, and $20(\simeq 48 \%)$ have an $A_{\mathrm{Li}}$ below that of the Hyades, while for 12 stars ( $\simeq 28 \%$ ) only an upper limit for $A_{\mathrm{Li}}$ could be measured.

The six stars we find above the Pleiades top envelope are likely bona-fide ZAMS stars (see also Fig. 1b, where we plot all stars which we consider to be very young). This is reflected by both their fast rotation and high $A_{\mathrm{Li}}$. Listed in order of decreasing $T_{\text {eff }}$ these stars are: HD $171488\left(A_{\mathrm{Li}}=3.1\right.$, 
$\left.v \sin i=45 \mathrm{~km} \mathrm{~s}^{-1}\right)$, HD $36869\left(A_{\mathrm{Li}}=3.3, v \sin i=30 \mathrm{~km} \mathrm{~s}^{-1}\right)$, HD $116402\left(A_{\mathrm{Li}}=3.1, v \sin i=35 \mathrm{~km} \mathrm{~s}^{-1}\right)$, HD 217343 $\left(A_{\mathrm{Li}}=3.0, v \sin i=12 \mathrm{~km} \mathrm{~s}^{-1}\right)$, HD $222259 \mathrm{~A}\left(A_{\mathrm{Li}}=3.1\right.$, $\left.v \sin i=17 \mathrm{~km} \mathrm{~s}^{-1}\right)$ and HD $202917\left(A_{\mathrm{Li}}=3.0, v \sin i=\right.$ $14 \mathrm{~km} \mathrm{~s}^{-1}$ ).

It is quite surprising that our sample includes also four apparently single MS stars with significant rotation and an $A_{\mathrm{Li}}$ much lower than that of the Hyades, and comparable to or lower than that of the Sun. Their low $A_{\mathrm{Li}}$ is not easily understood, and they deserve a separate discussion. Listed in order of decreasing $T_{\mathrm{eff}}$ these four stars are: HD $199672(v \sin i=$ $\left.11 \mathrm{~km} \mathrm{~s}^{-1}\right)$, HD $108361 \mathrm{~A}\left(v \sin i \leq 9 \mathrm{~km} \mathrm{~s}^{-1}\right)$, HD 184525 $\left(v \sin i=9 \mathrm{~km} \mathrm{~s}^{-1}\right)$ and HD $207377 \mathrm{~A}\left(v \sin i \leq 11 \mathrm{~km} \mathrm{~s}^{-1}\right)$. The last two are particularly intriguing because they are both chromospherically active (showing Ca II K emission line), and HD 184525 is also a bright $\mathrm{X}$-ray source. Hence, their very low $A_{\mathrm{Li}}$ is quite puzzling. HD $108361 \mathrm{~A}$ has no emission in the Ca II K line, while for HD 199672 we do not have a spectrum of the Ca II H\&K region.

These four stars are very interesting. They could be objects which suffered peculiar angular momentum histories, and they may represent the elusive population of young Lipoor stars predicted by rotational mixing induced models (e.g. Pinsonneault et al. 2000; Delyiannis et al. 2000). Considering that we do not expect to have any selection bias in our sample, apart from rotation, and making the assumption that all our single stars are due to a recent star formation burst, we can conclude that these young Li-poor stars represent much less than $\simeq 10 \%$ of the entire population. These objects would have to be born as extremely fast rotators (if rotational mixing was the mechanism responsible for Li depletion), considering that, even after a strong spin-down, they still maintain rotational velocities of the order of $10 \mathrm{~km} \mathrm{~s}^{-1}$.

To conclude, it is not obvious how to explain the low $A_{\mathrm{Li}}$ found in these four single rapidly rotating MS stars. They clearly deserve further investigation, in particular HD 184525. As it can be seen from Fig. 2a, there is a fifth star (HD $127352 \mathrm{~B}, \log T_{\text {eff }}=3.725, B-V=0.79$ ) with a very low $A_{\mathrm{Li}}$; but for such cool stars most of the $\mathrm{Li}$ is already depleted at the age of the Hyades, therefore the upper limit we found is not unsual even for a fast rotator.

Figure $2 \mathrm{~b}$ shows the $A_{\mathrm{Li}}$ vs. $\log T_{\text {eff }}$ distribution for the single stars above the MS. A large dispersion is present, but in this case the low $A_{\mathrm{Li}}$ which occurs for five stars, in order of decreasing $T_{\text {eff }}$, HD $20837 \mathrm{~B}\left(v \sin i=8 \mathrm{~km} \mathrm{~s}^{-1}\right)$, HD $68676(v \sin i=$ $\left.12 \mathrm{~km} \mathrm{~s}^{-1}\right)$, HD $84353\left(v \sin i=15 \mathrm{~km} \mathrm{~s}^{-1}\right)$, HD 101117 $\left(v \sin i=10 \mathrm{~km} \mathrm{~s}^{-1}\right)$ and HD $153926\left(v \sin i=11 \mathrm{~km} \mathrm{~s}^{-1}\right)$ is not surprising. If we compare our results with those of Mallik (1999), we see that in the restricted $T_{\text {eff }}$ range of our sample the two distributions are quite similar. Many surveys of evolved stars have shown a rather sharp decrease in $A_{\mathrm{Li}}$ in the subgiant phase just after the objects leave the MS (Randich et al. 2000; Lèbre et al. 1999). It is interesting to note that the drastic drop in $A_{\mathrm{Li}}$ after the MS has been associated with effects related to the stellar rotational history. In other words, it happens because the stars are slowed down from an initial high rotation (Talon \& Charbonnel 1998). However, the five stars mentioned above are still pretty fast rotators despite their low $A_{\mathrm{Li}}$.
Single evolved stars with enhanced rotation are rare and pose, until now, a difficult challenge for astrophysics. A dredgeup of angular momentum from a rapidly rotating deep interior to the stellar surface is hypothesized by different authors (e.g., Simon \& Drake 1989), but other hypotheses like the accretion of brown dwarfs or planets by giant stars can lead to significant spin-up of the stellar surface (Siess \& Livio 1999). Both scenarios are still open for observational test. For the five fast rotators discussed above these two scenarios may be hypothesized to explain their enhanced rotation. Nevertheless, because these stars show low $A_{\mathrm{Li}}$, an additional hypothesis seems necessary: if dredge-up of angular momentum did occur, one should not expect lithium production in the stellar interior; on the other hand, if accretion of brown dwarfs or planets is the origin of the observed high rotation, these objects have had no significant $A_{\mathrm{Li}}$. A crucial test for such a hypothesis would be, in the present case, an observation of CNO ratios combined with beryllium measurements.

Finally, Fig. 2b shows two stars, HD $106506(v \sin i=$ $\left.125 \mathrm{~km} \mathrm{~s}^{-1}\right)$ and HD $141943\left(v \sin i=38 \mathrm{~km} \mathrm{~s}^{-1}\right)$, with $A_{\mathrm{Li}}=3.3$. Such a high value of $A_{\mathrm{Li}}$ has never been found among evolved stars in the spectral range we are considering. As they also show extremely fast rotation, we consider these objects to be PMS stars. Their position in the $B-V$ vs. $M_{V}$ CMD is shown in Fig. $1 b$.

\subsection{The $A_{L i}$ vs. $\log T_{\text {eff }}$ diagram: close binaries}

Figure 3 shows the $A_{\mathrm{Li}}$ vs. $\log T_{\text {eff }}$ distribution for the close binaries (i.e. for components of SBs, including SBs that are members of VBs). Panels a and $b$ show the distribution of close binary components on (40 objects) and above ( 21 objects) the $\mathrm{MS}$, respectively.

As expected, the close binaries contain a higher proportion of stars with low $A_{\mathrm{Li}}$ than the single fast-rotating stars. In fact, high rotation can be sustained by tidal interaction also in old systems, which would otherwise have had enough time to destroy their Li. Thus, if one selects stars based on their high rotation rate, one may expect to select both young and old objects in the case of binaries. Moreover, we know that binarity can affect both $A_{\mathrm{Li}}$ and its links with rotation and activity (e.g. Randich et al. 1993, 1994; Fernandez-Figueroa et al. 1993; Barrado y Navascues et al. 1998), and that tidal effects are expected to influence $A_{\mathrm{Li}}$ in close binaries, which should retain more Li than single stars of the same age (Zahn 1994). For all these reasons it is not surprising that among the MS single components of SBs in our sample, $3(\simeq 8 \%)$ have an $A_{\mathrm{Li}}$ higher than that of the Pleiades, $16(\simeq 40 \%)$ have an $A_{\mathrm{Li}}$ between the Pleiades and Hyades, and $5(\simeq 12 \%)$ have an $A_{\mathrm{Li}}$ below that of the Hyades, while for 16 stars $(\simeq 40 \%)$ only an upper limit to $A_{\mathrm{Li}}$ could be determined.

The distribution of $A_{\mathrm{Li}}$ in binaries is different from that in single stars: more Li-poor objects are found in the binaries. The three stars above the Pleiades upper envelope are the binary components HD $202908 \mathrm{Ab}\left(A_{\mathrm{Li}}=3.2, v \sin i=10 \mathrm{~km} \mathrm{~s}^{-1}\right)$, HD $84323 \mathrm{a}\left(A_{\mathrm{Li}}=3.1, v \sin i=32 \mathrm{~km} \mathrm{~s}^{-1}\right)$ and HD $13183 \mathrm{a}$ $\left(A_{\mathrm{Li}}=3.1, v \sin i=22 \mathrm{~km} \mathrm{~s}^{-1}\right)$. While HD $84323 \mathrm{a}$ and 


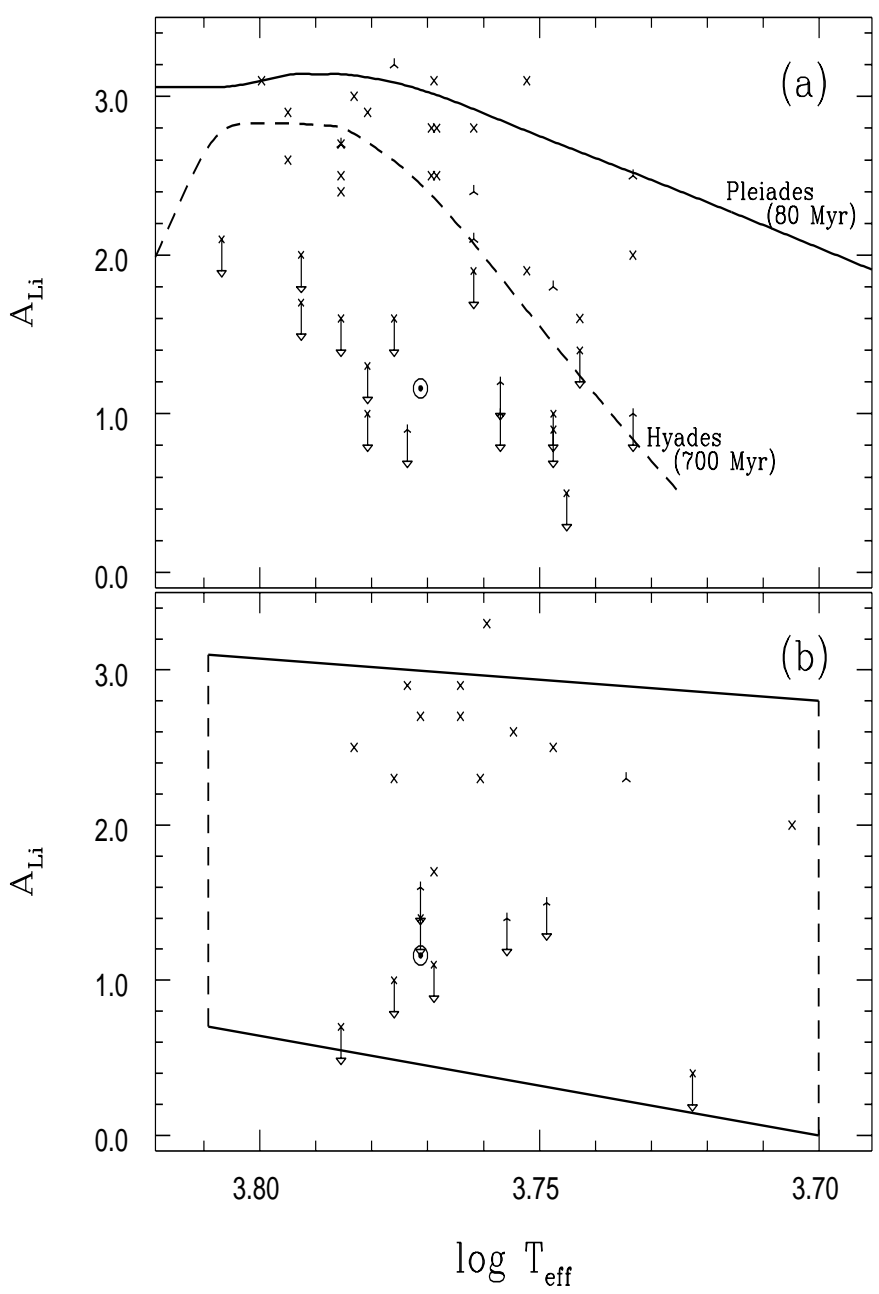

Fig. 3. $A_{\mathrm{Li}}$ vs. $\log T_{\text {eff }}$ for the close binaries. Symbols as in Fig. 1. Panel a) MS stars; the solid and dashed curves are the fiducial $A_{\mathrm{Li}}$ vs. $\log T_{\text {eff }}$ curves for the Pleiades (top envelope) and Hyades, respectively (adapted from Deliyannis 2000). Panel b) stars above the MS; the area inside the quadrangle is the region populated by subgiants, giants and supergiants (adapted from Mallik 1999). The Sun's position is shown in both panels.

HD 13183 a are likely to be ZAMS binaries (see their position in Fig. 1b), the classification of HD 202908 is more difficult. In fact, HD $202908 \mathrm{Ab}$ is part of a multiple system and is itself a VB whose primary component is an SB2 system (see Table 1 and Appendix 2 in Paper I). The $A_{\mathrm{Li}}$ values we computed for HD 202908 are $3.0\left(v \sin i=10 \mathrm{~km} \mathrm{~s}^{-1}\right)$, $3.2\left(v \sin i=10 \mathrm{~km} \mathrm{~s}^{-1}\right)$ and $2.4\left(v \sin i=9 \mathrm{~km} \mathrm{~s}^{-1}\right)$ for the $\mathrm{Aa}, \mathrm{Ab}$ and $\mathrm{B}$ component, respectively. This system deserves further investigation and its evolutionary status is not clear at this time. Finally, between the non-MS binaries (Fig. 3b) there is one, HD 104467, with $A_{\mathrm{Li}}=3.3$ and $v \sin i=23 \mathrm{~km} \mathrm{~s}^{-1}$, which we believe to be a PMS binary star (see also Fig. 1b).

\section{3. $A_{L i}$ vs. $v \sin i$}

The behavior of $A_{\mathrm{Li}}$ as a function of the projected rotational velocity $v \sin i$ is presented in Figs. $4 \mathrm{a}, \mathrm{b}$ for both single and binary stars. The first interesting feature that stands out is the large spread in the values of $A_{\mathrm{Li}}$ for stars with rotation lower than about $18 \mathrm{~km} \mathrm{~s}^{-1}$ : their $A_{\mathrm{Li}}$ show a spread of about 3 orders of magnitude, ranging from 0.4 dex to 3.0 dex for both single and binary stars. However, for single stars the spread decreases with increasing rotation, and the very fast rotators have all high $A_{\mathrm{Li}}$. In particular, all single MS stars rotating faster than about $15 \mathrm{~km} \mathrm{~s}^{-1}$ have $A_{\mathrm{Li}}>2.3$, and all single evolved stars rotating faster than about $18 \mathrm{~km} \mathrm{~s}^{-1}$ have $A_{\mathrm{Li}}>2.0$. No single MS star with $A_{\mathrm{Li}}<1.9$ is observed for $v \sin i>14 \mathrm{~km} \mathrm{~s}^{-1}$.

These results seem to indicate that another stellar parameter plays a significant role in the connection between rotation and $A_{\mathrm{Li}}$. This parameter seems to be important only for single solar-type stars with $v \sin i$ smaller that about $18 \mathrm{~km} \mathrm{~s}^{-1}$, while for higher rotation rates $A_{\mathrm{Li}}$ is always very high. These results are also found for evolved solar-type stars (de Medeiros et al. 2000; do Nascimento et al. 2000). We note that HD 106506 ( $v \sin i \simeq 125 \mathrm{~km} \mathrm{~s}^{-1}, A_{\mathrm{Li}}=3.3$ ) is not included in Fig. 4a not to compress the dynamic range of the figure too much. For both MS and evolved binary stars, as shown in Fig. 4b, the decrease of the spread in the $A_{\mathrm{Li}}$ distribution at high rotation rates is less marked than for single stars. Again this can be explained by the effect of tidally locked rotation: very old binaries can be strongly depleted in lithium while still maintaining a high rotation rate.

Pasquini et al. (1994) found a clear tendency for chromospherically active solar-type stars to have high $A_{\mathrm{Li}}$ and vice versa, despite the large scattering observed in the diagram of $A_{\mathrm{Li}}$ vs. Ca II surface flux. Similar results are found in samples of X-ray or EUV selected active stars (Favata et al. 1993, 1995; Tagliaferri et al. 1994, 2000; Jeffries 1995). The dependence of $A_{\mathrm{Li}}$ on stellar activity is consistent with the predictions of standard evolutionary models, according to which $A_{\mathrm{Li}}$ in MS stars should depend on stellar temperature, metallicity and age. Here, stellar activity is considered a good indicator of age for solar-type stars (e.g. Soderblom et al. 1991; Pizzolato et al. 2000). What is not clear is the physical cause of the large spread of the $A_{\mathrm{Li}}$ values for single stars with $v \sin i<18 \mathrm{~km} \mathrm{~s}^{-1}$.

Of course, one should expect that for some of these stars the low rotation rate reflects the $\sin i$ effect, i.e. stars seen poleon. If the stars with $v \sin i<18 \mathrm{~km} \mathrm{~s}^{-1}$ and high $A_{\mathrm{Li}}$ are all seen pole-on, this could reduce the observed spread somewhat. But it is difficult to believe that all the Li-rich slow-rotating stars analysed in this work should have such a preferred orientation of their axes.

One possible explanation is that the observed spread was produced during early MS lifetime. We recall that our sample is not homogeneous in age, and that substantial depletion occurs in G-type stars between the ZAMS and the Hyades age (Pasquini 2000). The observations of young open clusters show that cool $\left(T_{\mathrm{eff}}<5300 \mathrm{~K}\right) \mathrm{MS}$ stars present a clear spread of $A_{\mathrm{Li}}$ (Soderblom et al. 1994; Garcia Lopez et al. 1994; Randich et al. 1998) and that the fast rotators (i.e. stars rotating faster that $\sim 15 \mathrm{~km} \mathrm{~s}^{-1}$ ) are all close to the upper envelope of the $A_{\mathrm{Li}}$ distribution, while among the slower rotators a large spread is observed. Such a spread among the slower rotators has been discussed in the framework of the time needed to dissipate circumstellar disks during the PMS phase and its consequences on the stellar momentum evolution and the associated mixing in 


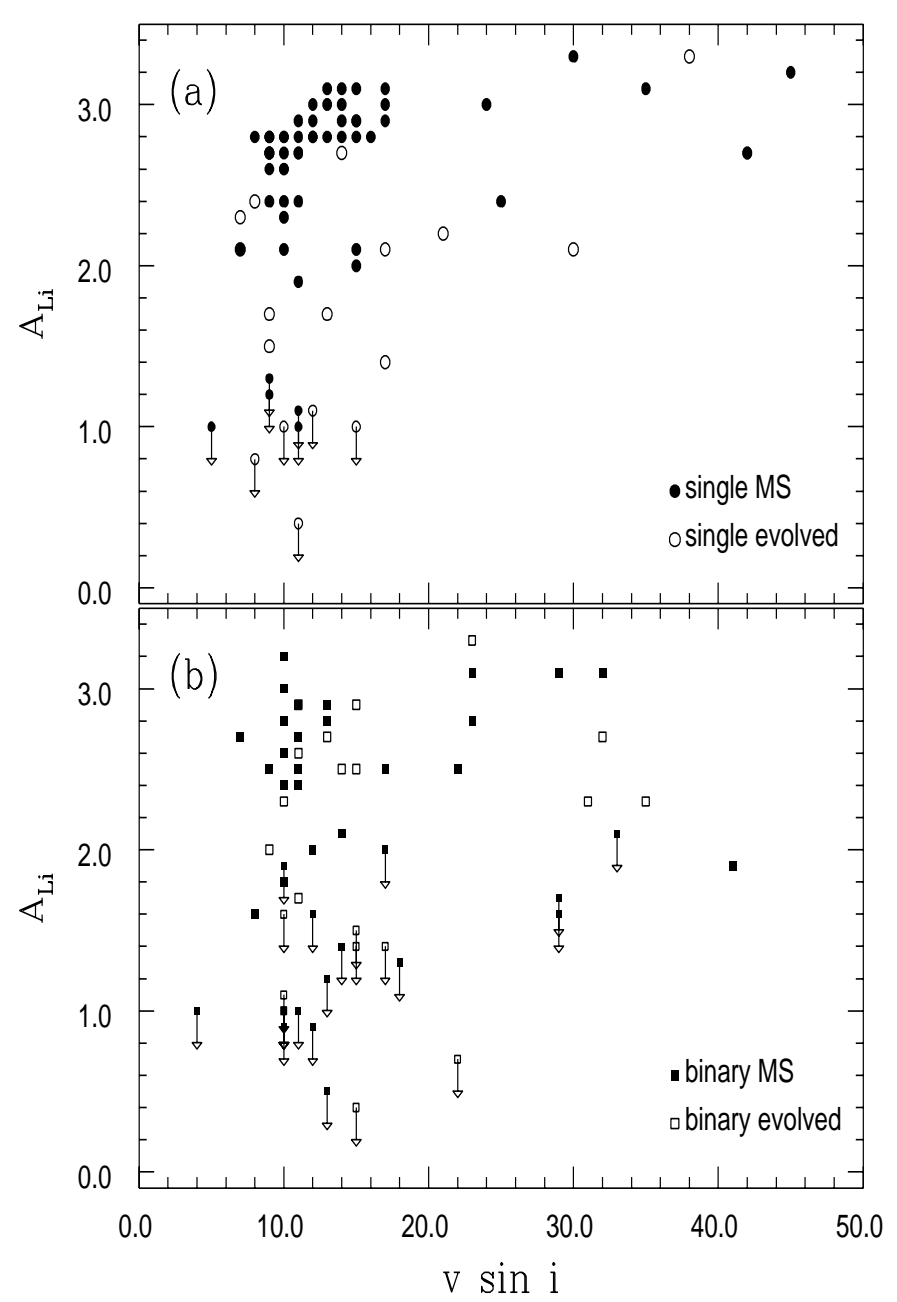

Fig. 4. $A_{\mathrm{Li}}$ vs. $v \sin i$ for single a) and binary stars b). Note that while all single stars with $v \sin i>18 \mathrm{~km} \mathrm{~s}^{-1}$ have $A_{\mathrm{Li}} \geq 2.0$, there are binaries with $v \sin i \sim 30 \mathrm{~km} \mathrm{~s}^{-1}$ for which only an upper limit to $A_{\mathrm{Li}}$ was obtained. In our sample of fast rotators, single stars have, on average, higher $A_{\mathrm{Li}}$ values than binary stars.

the early MS phase (Randich et al. 1998). However, the observations of young open clusters show no evidence for scattering among the hottest G-type stars, and these are the objects forming the bulk of our sample (in fact only a few of our stars are as cool as $5300 \mathrm{~K}$ ). Finally, we recall that Jones et al. (1999) have speculated that extra mixing on the MS could be driven by spin-down and angular momentum loss. If this is indeed true, the observed scattering in the $A_{\mathrm{Li}}$ versus $v \sin i$ diagram could be due to different initial rotation rates.

This document resulted from a POST operation and has expired from the cache. If you wish you can repost the form data to recreate the document by pressing the reload button.

\section{4. $L_{x}$ vs. $v \sin i$}

Solar type stars with moderate to high rotation rates are expected also to be active X-ray sources. Thus we searched the ROSAT all sky survey catalog (RASS, Vöges et al. 1999) and, in case of detection, derived the ROSAT PSPC X-ray luminosity in the $0.2-2.5 \mathrm{keV}$ energy band (see Sect. 3.6 in Paper I).



Fig. 5. $L_{\mathrm{X}}$ vs. $v \sin i$ for the single a) and binary b) stars in our sample detected in the RASS. As expected, $L_{\mathrm{X}}$ correlates with $v \sin i$, although with a large scatter, and all fast rotators are also very active in X-rays (with the exception of a few stars that are not detected, see text).

We found that 81 out of the 110 solar-type stars we studied were detected in the RASS (note that in the X-ray data we cannot separate the contribution of the components of VBs or SBs; see Paper I). In particular, of the 25 stars in our sample with $v \sin i>18 \mathrm{~km} \mathrm{~s}^{-1}$, only three (10\%) are not detected: HD 73204, HD 92648 and HD 141710: two binary systems with F6V and F7/8V primary stars, respectively, and a single G1/2 III star (see Paper I). In contrast, of the 85 stars in our sample with $v \sin i<18 \mathrm{~km} \mathrm{~s}^{-1}$, only $59(\simeq 69 \%)$ are detected.

In Figs. 5a, b we plot $L_{\mathrm{X}}$ as a function of $v \sin i$. We note that among the single stars with $v \sin i>18 \mathrm{~km} \mathrm{~s}^{-1}$ (Fig. 5a), all the 10 stars detected, except one, have $L_{X}$ close to or larger than $5 \times 10^{29} \mathrm{erg} \mathrm{s}^{-1}$, a very large value for single stars. The exception is $\operatorname{HD} 136160\left(v \sin i=25 \mathrm{~km} \mathrm{~s}^{-1} ; L_{\mathrm{X}}=1.8 \times 10^{29} \mathrm{erg} \mathrm{s}^{-1}\right)$, which, among single stars, has the earliest (F6V) spectral type in our sample and moderately slow rotation for that spectral type. The well-established correlation between $L_{X}$ and rotation rate (Pallavicini et al. 1981; Schmitt et al. 1985; Hempelmann et al. 1995; Stauffer et al. 1997) is clearly observed. There also seems to be a sort of saturation limit, which would appear even more clearly if we had included HD 106506, which has 


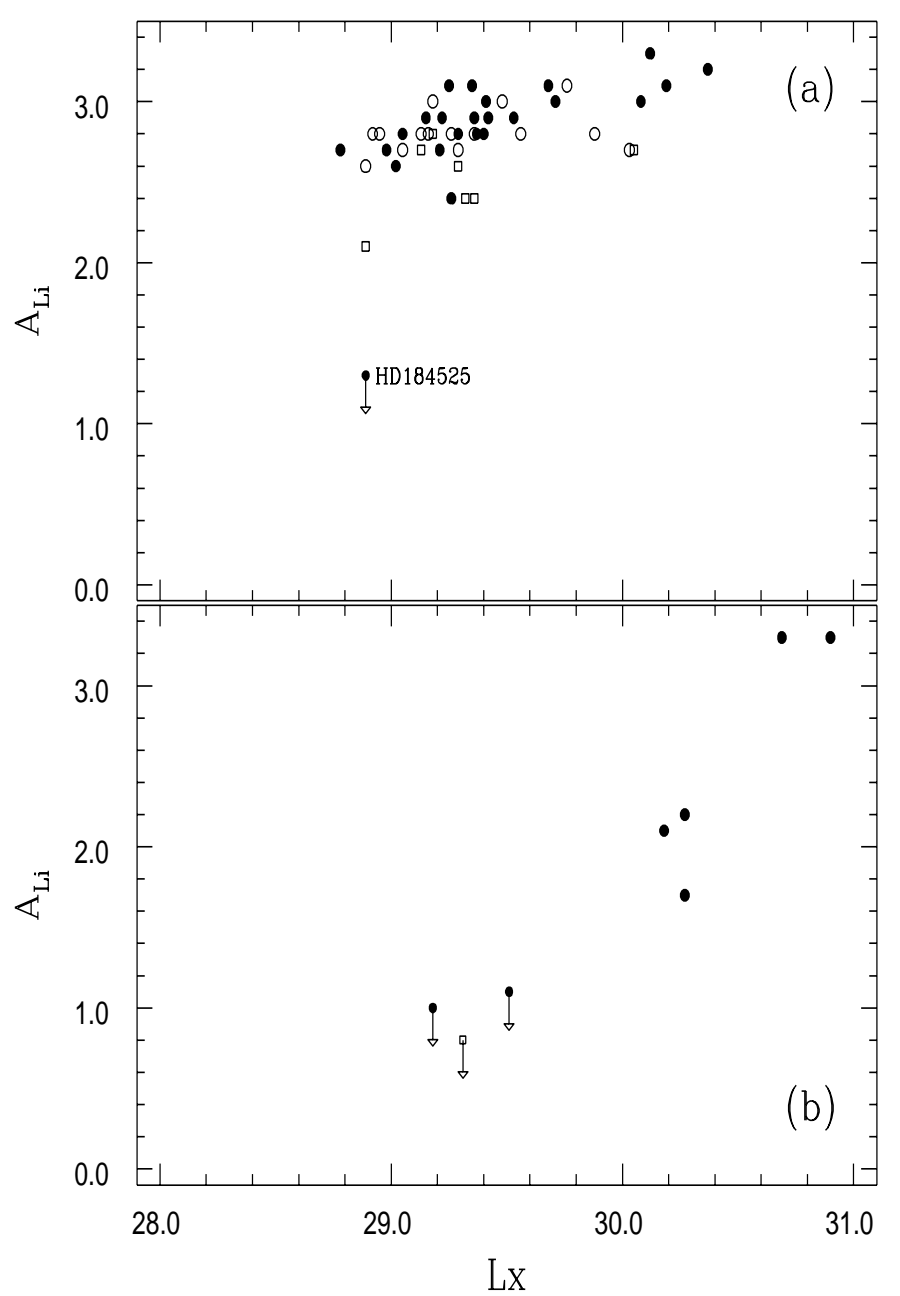

Fig. 6. $L_{\mathrm{X}}$ vs. $A_{\mathrm{Li}}$ for single stars. Symbols are as in Fig. 1. Panel a) stars on the main sequence; Panel b) stars above the main sequence. $L_{\mathrm{X}}$ is clearly correlated with $A_{\mathrm{Li}}$. The correlation is particularly prominent for non-MS stars (panel b)), although the latter sample is rather small and the correlation is dominated by the two PMS candidates.

$v \sin i \simeq 125 \mathrm{~km} \mathrm{~s}^{-1}$ and $L_{\mathrm{X}}=8 \times 10^{30} \mathrm{erg} \mathrm{s}^{-1}$ (we have omitted this star in order to obtain a reasonable dynamic range of the figures and preserve clarity). As expected, in particular at saturation level, single evolved stars have larger $L_{\mathrm{X}}$ compared to single MS stars for similar values of $v \sin i$.

Among the binary stars (Fig. 5b) with $v \sin i>18 \mathrm{~km} \mathrm{~s}^{-1}$ all the 13 stars detected, except two, have an $L_{X}$ near or larger than $8 \times 10^{29} \mathrm{erg} \mathrm{s}^{-1}$. The exceptions are HD 218602, an SB1 $\left(\mathrm{F} 9 \mathrm{~V}+\right.$ ?) system with $L_{\mathrm{X}}=3 \times 10^{29} \mathrm{erg} \mathrm{s}^{-1}$ and HD 81997 , an F5V + K5V binary system with $L_{\mathrm{X}}=1.4 \times 10^{29} \mathrm{erg} \mathrm{s}^{-1}$. The $L_{\mathrm{X}}$ vs. $v \sin i$ correlation is clearly observed also for binary stars. The $L_{X}$ distributions for MS and evolved binaries are similar, although the largest $L_{\mathrm{X}}$ are observed for evolved binaries. The spread seen for $v \sin i<18 \mathrm{~km} \mathrm{~s}^{-1}$ could be partially due, for both single and binary stars, to the presence of a solar-like activity cycle, which in case of the Sun causes $L_{X}$ to vary by more than a dex (Peres et al. 2000).

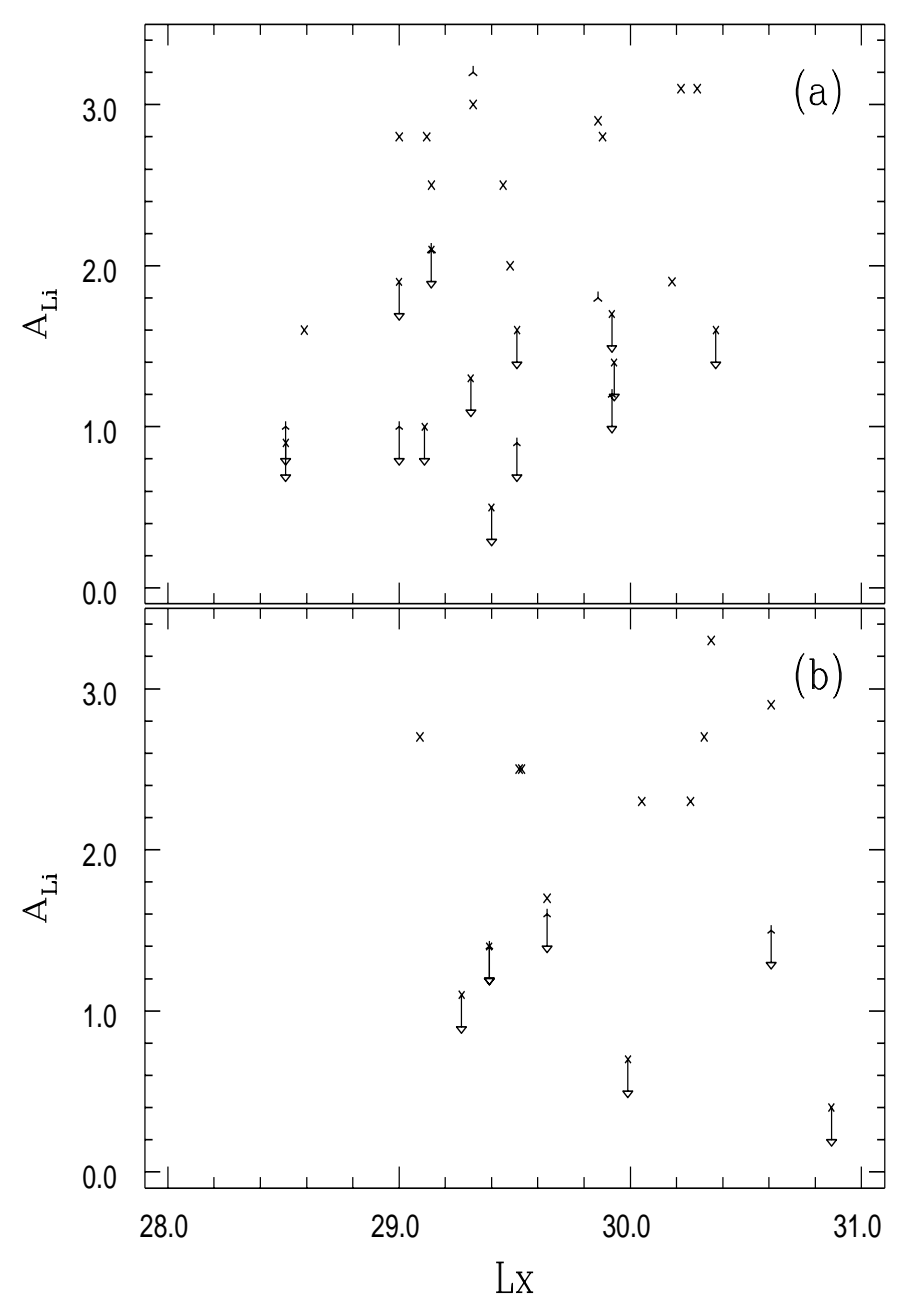

Fig. 7. $L_{\mathrm{X}}$ vs. $A_{\mathrm{Li}}$ for the binary sample. Symbols as in Fig. 1. Panel a) MS stars; Panel b) stars above the main sequence. No correlation is apparent.

\section{5. $L_{X} v s . A_{L i}$}

The behavior of $A_{\mathrm{Li}}$ as a function of $L_{\mathrm{X}}$ is displayed in Figs. 6a, b. Despite the lower number of stars in our sample which have both $A_{\mathrm{Li}}$ and $L_{\mathrm{X}}$ measured, one observes that for single stars (Fig. 6a) there is a clear tendency for a correlation between $A_{\mathrm{Li}}$ and $L_{\mathrm{X}}$. The only star in Fig. 6a with a very low $A_{\mathrm{Li}}$ is HD 184525 , which we have already found to be a peculiar object (see Sect. 3.1). Such a correlation between $A_{\mathrm{Li}}$ and coronal activity in solar-type stars is also reported by other authors (Tagliaferri et al. 1994; Favata et al. 1995; Jeffries 1995).

It is clear from Figs. 7a,b that no correlation between $A_{\mathrm{Li}}$ and $L_{\mathrm{X}}$ exists for binary stars. This is an interesting, but not surprising result, because old binaries, which have suffered strong Li depletion, can still sustain high activity levels through high, tidally induced rotation rates. In contrast, the single fast-tomoderately rotating nearby solar-type stars show a definitive tendency for a correlation between $A_{\mathrm{Li}}$ and rotation, as well as between $A_{\mathrm{Li}}$ and $L_{\mathrm{X}}$. We conclude that all single unevolved solar type stars with $v \sin i>18 \mathrm{~km} \mathrm{~s}^{-1}$ are strong X-ray emitters and have high $A_{\mathrm{Li}}$. Most likely, they are all very young objects, either ZAMS or PMS. 


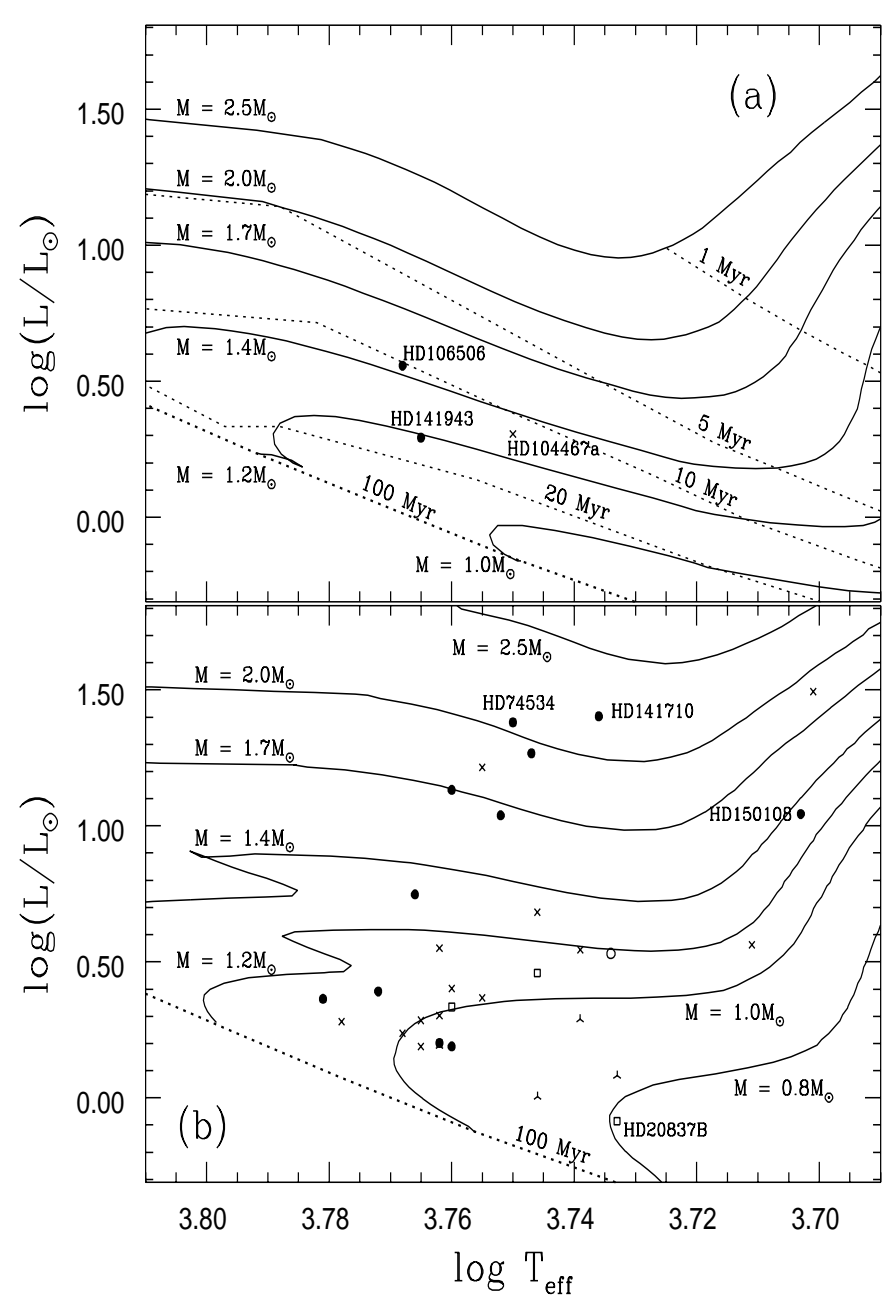

Fig. 8. $\log \left(L / L_{\odot}\right)$ vs. $\log T_{\text {eff }}$ diagram for the PMS and evolved stars of our sample. Symbols are as in Fig. 1. Panel a) shows the stars believed to be PMS objects as well as evolutionary tracks (continuous lines) and isochrones (dotted lines) for PMS stars (adapted from D'Antona \& Mazzitelli 1997). Panel b) shows the stars found to lie above the upper limit of the Hipparcos MS (see Fig. 1) as well as evolutionary tracks (continuous lines) and the $100 \mathrm{Myr}$ isochrone (dotted line) for evolved stars (adapted from Ventura et al. 1998).

\subsection{Masses and evolutionary status}

We have roughly estimated masses and ages for the PMS and evolved stars in our sample. For the PMS stars we used the evolutionary tracks and isochrones of D'Antona \& Mazzitelli (1997), for the evolved stars we used those by Ventura et al. (1998). In both cases, we corrected for the different temperature scales and/or other systematic errors by forcing the $100 \mathrm{Myr}$ isochrone to fit the Hipparcos MS. The resulting shift in $\log \left(L / L_{\odot}\right)$ of the models, adopting the Hipparcos $M_{V}$ and bolometric corrections, was about $-0.2 \mathrm{dex}$.

Figure 8a shows the stars we believe to be PMS objects, and Fig. $8 \mathrm{~b}$ the stars we found to lie beyond the upper limit of the Hipparcos MS (see Fig. 1). The PMS stars have ages in the 10-18 Myr interval and masses in the 1.4-1.2 $M_{\odot}$ interval. HD 106506, which is the fastest rotator in our sample, is also found to be the youngest and the most massive star among the PMS objects. Among the evolved stars HD 141710 and HD 74534, with masses of about $2.2 M_{\odot}$ and $2 M_{\odot}$, respectively, are the most massive stars in our sample. Their estimated ages are $<0.9 \mathrm{Gyr}$ and $<2 \mathrm{Gyr}$, respectively. Among the most evolved single stars we find HD 150108 which has $M \simeq 1.35 M_{\odot}$ and an age $\simeq 4 \mathrm{Gyr}$. With an age $>10 \mathrm{Gyr}$, HD $20837 \mathrm{~B}\left(M \simeq 0.8 M_{\odot}\right)$ is the oldest in our sample of evolved stars.

We have tried to detect any correlation between the stellar mass and the residuals of the individual stars from the correlations discussed above. None was found, showing that the observed surface activity is much more strongly controlled by the rotation and magnetic field in the stellar envelope than by the interior structure, which is primarily dependent on the mass of the star.

\section{Conclusion}

In this paper, we have discussed the results of high-resolution spectroscopy and high-precision photometry of a sample of 110 nearby solar-type stars (42 single stars, $33 \mathrm{VBs}$, and $35 \mathrm{SBs}$ ), selected for their fast rotation from a CORAVEL radial-velocity survey of late-F to G-type stars brighter than $V \simeq$ 8.6. Counting single stars, single components of VBs (which are treated as single stars), plus SB components with inferred spectral classifications, we discussed results for a total of 138 objects (77 single stars and 61 close binary components). Of these, 99 are MS stars (59 single and 40 components of close binaries) and the remaining 39 are evolved stars (18 single and 21 components of close binaries).

The single stars of our sample show statistically higher $A_{\mathrm{Li}}$ and activity level than unbiased samples of nearby field stars with similar spectral types. In particular, among the MS single stars of our sample we find that about $10 \%$ of them have an $A_{\mathrm{Li}}$ higher than that of the Pleiades, while about $64 \%$ have $A_{\mathrm{Li}}$ between that of the Pleiades and Hyades. Quite surprisingly, we also find four rapidly-rotating single MS stars with very low $A_{\mathrm{Li}}$. These four stars could represent examples of stars which suffered peculiar angular momentum histories, but it is not clear how to explain the observed characteristics. These stars deserve further investigation.

The distribution of $A_{\mathrm{Li}}$ for single evolved stars is characterized by a large spread, as already observed by other authors for evolved field stars. We find five evolved stars with very low $A_{\mathrm{Li}}$ that are still rather fast rotators. Their low $A_{\mathrm{Li}}$ cannot be explained by effects related to the stellar rotational history, and these stars also deserve further detailed investigation. We finally identify two new single PMS stars.

The MS binaries of our sample show a larger fraction of stars with low $A_{\mathrm{Li}}$. Only $8 \%$ of the binaries have $A_{\mathrm{Li}}$ higher than that of the Pleiades, while about $40 \%$ have $A_{\mathrm{Li}}$ between that of the Pleiades and Hyades. This is not surprising, as high stellar rotation can be maintained by tidal interaction also in old binaries which have had enough time to destroy their Li. Thus, in the case of binaries our selection criteria allow both young and old systems to be selected. We also find one new PMS binary star.

The behaviour of $A_{\mathrm{Li}}$ as a function of $v \sin i$ for single stars is characterized by a large scattering in $A_{\mathrm{Li}}$ for rotations lower than about $18 \mathrm{~km} \mathrm{~s}^{-1}$. The scattering decreases with 
increasing rotation, and the single very fast rotators are all stars with high $A_{\mathrm{Li}}$. These results seem to indicate that another stellar parameter plays a relevant role for the rotation- $A_{\mathrm{Li}}$ connection in single solar-type stars with $v \sin i<18 \mathrm{~km} \mathrm{~s}^{-1}$. Although the physical cause of the large scattering is not clear, it could reflect different initial rotation rates. For both MS and evolved binaries the spread of $A_{\mathrm{Li}}$ remains large also at high rotation rates. This is because even very old close binaries can maintain a high $v \sin i$ through tidal synchronization.

We have also searched the ROSAT All Sky Survey cata$\log$ and derived the PSRC X-ray luminosity for the stars detected. We find that 81 of the 110 possible stars were detected in the RASS (for X-ray data we cannot separate the contribution of VBs and SBs components). Of the 25 stars in our sample with $v \sin i>18 \mathrm{~km} \mathrm{~s}^{-1}$ only three are not detected. Out of the 85 stars with $v \sin i<18 \mathrm{~km} \mathrm{~s}^{-1}, 59$ are detected. The well-established correlation between $L_{\mathrm{X}}$ and $v \sin i$ is thus clearly observed, in particular for single stars. There also seems to be a kind of saturation limit.

Our results, in agreement with recent findings from EUV and $\mathrm{X}$-ray surveys, confirm the presence of young very active stars close to the Sun, although our sample has less extreme characteristics than the samples so far selected in the EUV and $\mathrm{X}$-ray bands at the current flux limits (essentially the ROSAT all-sky survey limits).

Acknowledgements. Stellar activity research at INAF-Catania Astrophysical Observatory is supported by the Italian MIUR ("Ministry for Education, Universities and Research") and by the Regional Government of Sicily ("Regione Sicilia"). Stellar activity research at INAF-Brera Astronomical Observatory is supported by the Italian MIUR and by ASI ("Italian Space Agency"). Stellar activity research at the University Federal of Rio Grande do Norte is partially supported by the CNPq brazilian agency. The programmes on Galactic structure at Copenhagen University are financially supported by the Danish Natural Science Research Council and by Carlsberg Foundation. This research has made use of the SIMBAD database, operated at CDS, Strasbourg, France. Special thanks are due to the ESO staff for the collaboration and technical support during the observations, to Dr. Francesca D'Antona (INAF - Rome Astronomical Observatory) and Dr. Bjarne R. Jørgensen (Lund Observatory) for useful discussions. We also thank the referee, Dr. R. Garcia-Lopez, for his useful comments and Ms. Luigia Santagati for revision of the manuscript.

\section{References}

Barrado y Navascues, D., De Castro, E., Fernandez-Figueroa, M. J., Cornide, M., \& Garcia Lopez, R. J. 1998, A\&A, 337, 739

Charbonnel, C., Deliyannis, C. P., \& Pinsonneault, M. H. 2000, in The Light Elements and their Evolution, ed. L. da Silva, R. de Medeiros, \& M. Spite, IAU Symp., 198, 87

Cutispoto, G. 2002, AN, 323, 325

Cutispoto, G., Pastori, L., Pasquini, L., et al. 2002, A\&A, 384, 491 (Paper I)

Deliyannis, C. P. 2000, in Stellar Clusters and Associations, ed. R. Pallavicini, G. Micela, \& S. Sciortino, ASP Conf. Ser., 198, 235

Deliyannis, C. P., Pinsonneault, M. H., \& Charbonnel, C. 2000, in The Light Elements and their Evolution, ed. L. da Silva, R. de Medeiros, \& M. Spite, IAU Symp., 198, 61
D’Antona, F., \& Mazzitelli, I. 1997, Mem. Soc. Astron. It., 68, 807

de Medeiros, J. R., do Nascimento, J. D., Sankarankutty, S., Costa, J. M., \& Maia, M. R. G. 2000, A\&A, 363, 239

do Nascimento, J. D., Charbonnel, C., Lebre, A., de Laverny, P., \& de Medeiros, J. R. 2000, A\&A, 357, 931

Favata, F., Barbera, M., Micela, G., \& Sciortino, S. 1993, A\&A, 277, 428

Favata, F., Barbera, M., Micela, G., \& Sciortino, S. 1995, A\&A, 295, 147

Fernandez-Figueroa, M. J., Barrado, D., de Castro, E., \& Cornide, M. 1993, A\&A, 274, 373

Garcia Lopez, R. J., Rebolo, R., \& Martin, E. L. 1994, A\&A, 282, 518

Hempelmann, A., Schmitt, J. H. M. M., Schultz, M., Ruedinger, G., \& Stepien, K. 1995, A\&A, 294, 515

Houk, N., Swift, C. M., Murray, C. A., Penston, M. J., \& Binney, J. J. 1997, in Proc. Hipparcos Venice 97 Symp., ESA SP-402, 279

Jeffries, R. D. 1995, MNRAS, 273, 559

Jones, B. F., Fisher, D., \& Soderblom, D. R. 1999, AJ, 117, 330

Lèbre, A., de Laverny, P., de Medeiros, J. R., Charbonnel, C., \& da Silva, L. 1999, A\&A, 345, 936

Mallik, S. V. 1999, A\&A, 352, 495

Nordström, B., Andersen, J., Olsen, E. H., et al. 1999, Ap\&SS, 265, 235

Pallavicini, R., Golub, L., Rosner, R., et al. 1981, ApJ, 248, 279

Pasquini, L. 2000, in The Light Elements and their Evolution, ed. L. da Silva, R. de Medeiros, \& M. Spite, IAU Symp., 198, 269

Pasquini, L., Liu, Q., \& Pallavicini, R. 1994, A\&A, 287, 191

Pasquini, L., Randich, S., \& Pallavicini, R. 1997, A\&A, 325, 535

Peres, G., Orlando, S., Reale, F., Rosner, R., \& Hudson, H. 2000, ApJ, 528,537

Pinsonneault, M. H., Charbonnel, C., \& Deliyannis, C. P. 2000, in The Light Elements and their Evolution, ed. L. da Silva, R. de Medeiros, \& M. Spite, IAU Symp., 198, 74

Pizzolato, N., Maggio, A., \& Sciortino, S. 2000, A\&A, 361, 614

Randich, S. 2001, A\&A, 377, 512

Randich, S., Gratton, R., \& Pallavicini, R. 1993, A\&A, 273, 194

Randich, S., Gianpapa, M. S., \& Pallavicini, R. 1994, A\&A, 283, 893

Randich, S., Martin, E. L., Garcia Lopez, R. J., \& Pallavicini, R. 1998, A\&A, 333, 591

Randich, S., Pasquini, L., \& Pallavicini, R. 2000, A\&A, 356, L25

Schmitt, J. H. M. M., Golub, L., Harnden, F. R. Jr., et al. 1985, ApJ, 290, 30

Siess, L., \& Livio, M. 1999, MNRAS, 308, 1133

Simon, T., \& Drake, S. A. 1989, ApJ, 346, 303

Stauffer, J. R., Hartmann, L. W., Prosser, C. F., et al. 1997, ApJ, 479, 776

Soderblom, D. R., Duncan, D. K., \& Johnson, D. R. H. 1991, ApJ, 355,722

Soderblom, D. R., Jones, B. F., Balachandran, S., et al. 1993, AJ, 106, 1059

Tagliaferri, G., Cutispoto, G., Pallavicini, R., Randich, S., \& Pasquini, L. 1994, A\&A, 285, 272

Tagliaferri, G., Pastori, L., Cutispoto, G., \& Pallavicini, R. 2000, in The Light Elements and their Evolution, ed. L. da Silva, R. de Medeiros, \& M. Spite, IAU Symp., 198, 366

Talon, S., \& Charbonnel, C. 1998, A\&A, 335, 959

Ventura, P., Zeppieri, A., Mazzitelli, I., \& D’Antona, F. 1998, A\&A, 334, 953

Vöges, W., Aschenbach, B., Boller, Th., et al. 1999, A\&A, 349, 389

Zahn, J.-P. 1994, A\&A, 288, 829 